\title{
Adaptive Rollover Prevention for Automotive Vehicles with Differential Braking *
}

\author{
Selim Solmaz ${ }^{*}$, Mehmet Akar**, and Robert Shorten* \\ *Hamilton Institute, Nat. Univ. of Ireland-Maynooth, Co. Kildare (Tel:+353 \\ 1 7086100; Fax: +3531 7086269; e-mail: \\ selim.solmaz@nuim.ie,robert.shorten@nuim.ie). \\ ** Dept. of Electrical and Electronic Engineering, Boğaziçi University, \\ Istanbul, Turkey (e-mail:mehmet.akar@boun.edu.tr)
}

\begin{abstract}
In this paper we present an adaptive controller implementation based on the multiple models, switching, and tuning (MMST) paradigm [13, 14, 15] for preventing un-tripped rollover in automotive vehicles. Our approach relies on differential-braking to keep the value of the Load Transfer Ratio (LTR) below a threshold. We first employ multiple models to infer the unknown center of gravity height and the suspension parameters of the vehicle, which are subsequently used to switch to the corresponding rollover controller. The proposed multi-controller switched scheme is shown via numerical simulations to result in better performance than its fixed robust counterpart.
\end{abstract}

Keywords: Automotive control; Multiple models; Parameter estimation; Adaptive control; Switched controllers.

\section{INTRODUCTION}

It is a well known fact that vehicle center of gravity (CG) position plays an important role for the vehicle dynamics and the vehicle road handling behavior. Therefore, the effects of changes in the $\mathrm{CG}$ position, or the uncertainty in the knowledge of it, have to be considered for analyzing vehicle dynamics, and must be accounted for in designing active control systems for accident mitigation. However, the difficulty is that this unknown parameter is not directly measurable and it can vary significantly with changing passenger and loading configurations; such changes are the most severe in large passenger vehicles such as (Sport Utility Vehicles) SUVs.

It is an irrefutable fact that vehicles with a high center of gravity such as vans, pickups, and the highly popular SUVs are more prone to rollover accidents than other passenger vehicles. According to recent statistical data [1], light trucks (pickups, vans and SUVs) were involved in nearly $70 \%$ of all the rollover accidents in the USA, with SUVs alone responsible for almost $35 \%$ of this total. The fact that the composition of the current automotive fleet in the U.S. consists of nearly $36 \%$ pickups, vans and SUVs [2], along with the recent increase in the popularity of SUVs worldwide, makes rollover an important safety problem.

There are two distinct types of vehicle rollover: tripped and untripped. A tripped rollover commonly occurs when a vehicle slides sideways and digs its tires into soft soil or strikes an object such as a curb or guardrail. Driver induced un-tripped rollover can occur during typical driving situations and poses a real threat for top-heavy vehicles. Examples are excessive speed during cornering, obstacle avoidance and severe lane change maneuvers, where rollover occurs as a direct result of the lateral wheel forces induced during these maneuvers. In recent years,

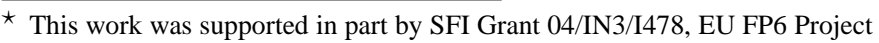
CEmACS 004175 and BAP 07HA202.
}

rollover has been the subject of intensive research, especially by the major automobile manufacturers, which is geared towards the development of rollover prediction schemes and occupant protection devices. It is however, possible to prevent such a rollover incident by monitoring the car dynamics and applying proper control effort ahead of time. Therefore there is a need to develop driver assistance technologies which would be transparent to the driver during normal driving conditions, while acting in emergency situations to recover handling of the vehicle until the driver recovers control of the vehicle [2].

The height of CG along with the lateral acceleration are the most important parameters affecting the rollover propensity of an automotive vehicle; while the vehicle lateral acceleration can be measured directly by sensors, the CG height can not be measured and it needs to be estimated indirectly. One such method for inferring $C G$ height was suggested in $[3,4,5]$. We utilize this result for the control strategy advocated in this paper with the aim to improve the performance of our active rollover mitigation systems. Specifically, we use multiple identification models for inferring the unknown vehicle CG height, which is then used to switch among a paired set of rollover prevention controllers.

Our motivation for considering an adaptive controller implementation is twofold. Firstly, adaptive controllers are the alternative option to the robust ones and they can potentially provide higher performance. Robust controllers have fixed gains that are chosen considering the worst-case that the plant undergoes; for the rollover problem the worst operating condition translates to operating the vehicle with the highest possible CG position. While choosing the controller gains for the worst-case guarantees the performance (i.e., safety) under the designed extreme operating condition, the feedback performance of the robustly controlled systems under less severe or even normal operating conditions are suboptimal. The second motivation for considering the adaptive feedback design for the rollover prevention 

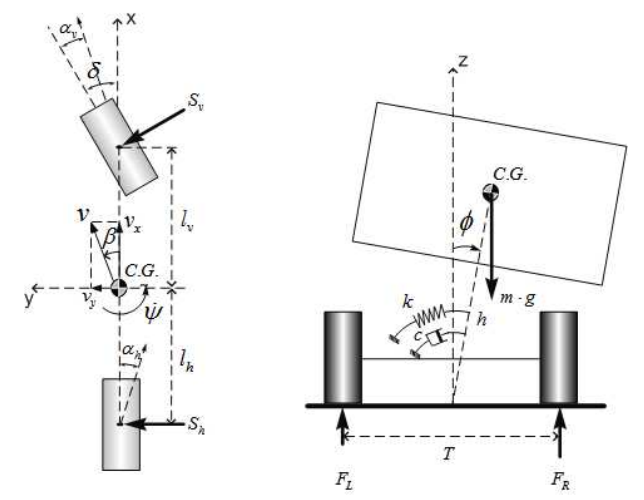

Fig. 1. Single track model with roll degree of freedom.

problem is related to the time constant of rollover accidents, which is on the order of seconds (sometimes even a fraction of a second) and is usually accepted to be quite small (see for example [6] for a discussion of this). While conventional adaptive controllers are known to have slow convergence rates and large transient control errors when the initial parameter errors are large (a factor that renders these control approaches unsuited for use in rollover mitigation applications), utilization of MMST type algorithms [7] may overcome these problems and provide high performance adaptive controllers. Therefore, when improving the controller performance and speed for the rollover problem is considered, MMST framework becomes an ideal choice as it can provide a rapid identification of the unknown parameters as part of the closed loop implementation. This way we can rapidly switch to a controller that is optimal for the maneuver and the vehicle operating conditions, thus improving the overall safety of the vehicle without sacrificing its performance.

The controller design described in the sequel is based on differential braking actuators only, where each of the $n$ proposed switched controllers based on differential braking actuator has a linear feedback structure with a fixed gain matrix $K_{\eta}$, where $\eta \in\{1,2, \ldots, n\}$. The choice of the control actuator is motivated by the desire to aid the exposition of the multiple model switched control implementation, as the resulting controllers are of simple proportional type. Our controllers are designed to keep the peak magnitude of LTR less than one, which is the criterion for preventing rollover occurrence as it is equivalent to preventing one-side wheel lift off. We demonstrate the effectiveness of the resulting control design with numerical simulations.

\section{VEHICLE MODELLING}

For the multiple model switched controller design that shall be described in the following sections, we utilize two separate vehicle models; the second order roll plane model, and the single track model with roll degree of freedom and with differential brake input. While we use the roll plane models for estimating the unknown CG height of the vehicle in real time, we utilize the single track model with roll degree of freedom to represent the vehicle in simulations and also to implement the output feedback controllers based on the lateral acceleration measurement and utilizing differential braking actuators. For detailed derivations of the following vehicle models see $[8,9]$.

Roll plane model: $\quad$ We use the roll plane model given here for the realtime estimation of CG height based on the multiple
Table 1. Model parameter definitions

\begin{tabular}{cll}
\hline Parameter & Description & Unit \\
\hline$\delta$ & Steering angle & {$[\mathrm{rad}]$} \\
$m$ & Vehicle mass & {$[\mathrm{kg}]$} \\
$v$ & Vehicle speed & {$[\mathrm{m} / \mathrm{s}]$} \\
$J_{x x}$ & Roll moment of inertia at CG & {$\left[\mathrm{kgm}^{2}\right]$} \\
$J_{z z}$ & Yaw moment of inertia at CG & {$\left[\mathrm{kgm}^{2}\right]$} \\
$l_{v}$ & Longitudinal CG position w.r.t. front axle & {$[\mathrm{m}]$} \\
$l_{h}$ & Longitudinal CG position w.r.t. rear axle & {$[\mathrm{m}]$} \\
$T$ & Vehicle track width & {$[\mathrm{m}]$} \\
$h$ & CG height from roll axis & {$[\mathrm{m}]$} \\
$c$ & Suspension roll damping coefficient & {$[\mathrm{Nms} / \mathrm{rad}]$} \\
$k$ & Suspension roll spring stiffness & {$[\mathrm{Nm} / \mathrm{rad}]$} \\
$C_{v}$ & Linear tire stiffness for front tire & {$[\mathrm{N} / \mathrm{rad}]$} \\
$C_{h}$ & Linear tire stiffness for rear tire & {$[\mathrm{N} / \mathrm{rad}]$} \\
$\beta$ & Sideslip angle at CG & {$[\mathrm{rad}]$} \\
$\dot{\psi}$ & Yaw rate & {$[\mathrm{rad} / \mathrm{s}]$} \\
$\phi$ & Roll angle & {$[\mathrm{rad}]$} \\
$\dot{\phi}$ & Roll rate & {$[\mathrm{rad} / \mathrm{s}]$} \\
\hline
\end{tabular}

model switching framework. The 2-state roll plane model is the simplest model capturing the roll dynamics of an automotive vehicle and it is free from the effects of uncertainties originating from unknown tire stiffness parameters; we emphasize that this a factor that makes the roll plane model suitable for the real time estimation of unknown CG position.

Under the small angles assumption, and with reference to right hand side of Figure 1, the equations of motion describing the roll plane dynamics can be expressed in the following $2^{\text {nd }}$ order state space form

$$
\left[\begin{array}{c}
\dot{\phi} \\
\ddot{\phi}
\end{array}\right]=\left[\begin{array}{cc}
0 & 1 \\
-\frac{k-m g h}{J_{x_{e q}}} & -\frac{c}{J_{x_{e q}}}
\end{array}\right] \cdot\left[\begin{array}{c}
\phi \\
\dot{\phi}
\end{array}\right]+\left[\begin{array}{c}
0 \\
\frac{m h}{J_{x_{e q}}}
\end{array}\right] a_{y},
$$

where $a_{y}$ is the lateral acceleration, and $g$ is the gravitational acceleration. As a simplifying assumption for the derivation of the model, it was assumed that relative to the ground, the sprung mass rolls about a fixed horizontal roll axis along the centerline of the vehicle body at the ground level. Also, $J_{x_{e q}}$ above denotes the equivalent roll moment of inertia of the vehicle about the roll axis, which is given by

$$
J_{x_{e q}}=J_{x x}+m h^{2} .
$$

For further description of the parameters appearing in the roll plane model refer to Table 1.

Single track model with roll degree of freedom: We use this model to represent the real vehicle in our simulations due to its relative simplicity. We note that this model is the simplest model with combined roll and lateral dynamics. With reference to Figure 1, we denote $\beta$ as the sideslip angle and $\dot{\psi}$ as the yaw rate of the vehicle representing vehicle motion on a horizontal surface. Then the equations of motion corresponding to this model are given as follows

$$
\left.\begin{array}{l}
\dot{x}=A x+B_{\delta} \delta+B_{u} u \\
a_{y}=v(\dot{\beta}+\dot{\psi}) \\
A=\left[\begin{array}{cccc}
-\frac{\sigma J_{x_{e q}}}{m J_{x x} v} & \frac{\rho J_{x_{e q}}}{m J_{x x} v^{2}}-1 & -\frac{h c}{J_{x x} v} & \frac{h(m g h-k)}{J_{x x} v} \\
\frac{\rho}{J_{z z}} & -\frac{\kappa}{J_{z z} v} & 0 & 0 \\
-\frac{h \sigma}{J_{x x}} & \frac{h \rho}{J_{x x} v} & -\frac{c}{J_{x x}} & \frac{m g h-k}{J_{x x}}
\end{array}\right] \\
B_{\delta}=\left[\begin{array}{lllll}
\frac{C_{v} J_{x e q}}{m J_{x x} v} & \frac{C_{v} l_{v}}{J_{z z}} & \frac{h C_{v}}{J_{x x}} & 0
\end{array}\right]^{T}, B_{u}=\left[\begin{array}{llll}
0-\frac{T}{2 J_{z z}} & 0 & 0
\end{array}\right]^{T}
\end{array}\right\}
$$




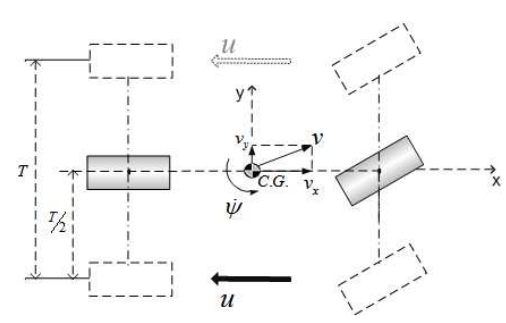

Fig. 2. Differential braking force as control input.

where $x=\left[\begin{array}{llll}\beta & \dot{\psi} & \dot{\phi} & \phi\end{array}\right]^{T}$ is the state, and $u$ represents the total effective differential braking force acting on the wheels; it is positive if braking is on the right wheels and negative if braking is on the left wheels. Differential braking force as the control input is depicted in Figure 2. Further notations and parameters appearing in (3) are described in Table 1.

In order to model the change in the vehicle longitudinal speed as a result of the braking force, we assume that the longitudinal wheel forces generated by the engine counteract the rolling resistance and the aerodynamic drag at all times. Under this assumption, the vehicle speed is approximately governed by

$$
\dot{v}=-\frac{|u|}{m} .
$$

Comment: The rationale for using differential braking actuators for the rollover mitigation problem discussed here is due to the fact that such actuators are already available in many modern production cars. Vehicles equipped with systems such as ABS (Anti-lock Braking System) and EBS (Electronic Brake System) or similar such systems are capable of selectively braking each of the wheels. Thus our design can potentially be implemented using these systems.

\subsection{The Load Transfer Ratio, LTR}

Traditionally, some estimate of the vehicle load transfer ratio $(L T R)$ has been used as a basis for the design of rollover prevention systems. The quantity $L T R$ [10] can be simply defined as follows

$$
L T R=\frac{\text { Load on Right Tires-Load on Left Tires }}{\text { Total Load }} .
$$

Clearly, $L T R$ varies within $[-1,1]$, and for a perfectly symmetric car that is driving straight, it is zero. The extrema are reached in the case of a wheel lift-off of one side of the vehicle, in which case $L T R$ becomes 1 or -1 depending on the side that lifts off. A dynamical approximation for the load transfer ratio, denoted $L T R_{d}$, is given as follows $[11,12]$

$$
L T R_{d}=-\frac{2(c \dot{\phi}+k \phi)}{m g T} .
$$

\section{ADAPTIVE ROLLOVER CONTROL DESIGN WITH MULTIPLE MODELS \& SWITCHING BASED ON DIFFERENTIAL BRAKING ACTUATORS}

In this section we describe an adaptive control design for preventing rollover based on differential braking actuators. We use a multiple switched controller structure where the controller switching logic is based on the real-time estimation of $\mathrm{CG}$ height $[3,4,5]$. We note that the adaptive control

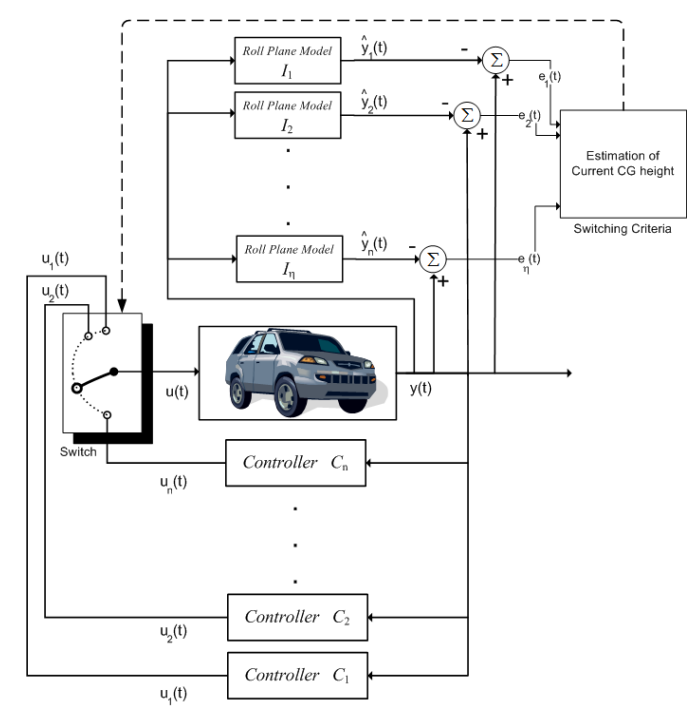

Fig. 3. Multiple model switched adaptive control structure.

implementation given here is inspired, at large, by the MMST control framework developed by Narendra et al. in a series of publications [7, 13, 14, 15]. In the MMST control framework, each identification model is paired-up with a controller, and based on a performance index of the identification errors a model/controller pair is chosen to control the plant at every instant.

Our multiple switched controller design is unique in the sense that it enables us to synthesize locally robust controllers corresponding to each CG height configuration. This approach, as compared to the alternative robust controller designs in literature, yields improved vehicle cornering performance. We emphasize that in this controller implementation, adaptation is a byproduct of the switching itself.

Since we wish to prevent rollover of the vehicle, our switched controllers are designed to keep the peak magnitude of the load transfer ratio less than one, which implies preventing one-side wheel lift-off, and thus avoiding rollover. The switched multiple model control structure is schematically shown in Figure 3, where there are $n$ identification models driven by the same plant output, which are paired up with $n$ locally robust state feedback controllers. In what follows, we first describe the switched identification algorithm as a control switching criterion, and then give the implementation of the switched adaptive rollover controller design utilizing differential braking actuators.

\subsection{Controller switching criteria}

The height of CG along with the lateral acceleration are the most important parameters affecting the rollover propensity of an automotive vehicle; while the vehicle lateral acceleration can be measured directly by sensors, the CG height can not be measured and it needs to be estimated indirectly. Here we use multiple identification models for inferring the unknown vehicle CG height along with the relevant suspension parameters in real time $[3,4,5]$, which is then used as a criterion to switch among a paired set of locally robust rollover prevention controllers. We emphasize that due to this structure of multiple indirect estimation models and the paired controllers, the suggested feedback implementation is an adaptive control approach for the problem of mitigation of rollover, which involves inherent parametric 
uncertainties due to the unknown and/or time varying vehicle parameters.

The identification models are based on the $2^{\text {nd }}$ order roll plane model (1) and are mainly used to determine the unknown CG height of the vehicle. The estimation models are obtained by varying the uncertain model parameters within bounded intervals and at a finite number of grid points, where the uncertain parameters are the CG height $h$, linear roll spring stiffness $k$, and the roll damping coefficient $c$. Specifically, each of the unknown parameters is assumed to belong to a closed uncertainty interval such that $h \in \mathscr{H}, k \in \mathscr{K}$, and $c \in \mathscr{C}$, where each interval contains a sufficient number of grid points so that they can be represented as $\mathscr{H}=\left\{h_{1}, h_{2}, h_{3}, \ldots, h_{p}\right\}, \mathscr{K}=$ $\left\{k_{1}, k_{2}, k_{3}, \ldots, k_{q}\right\}$, and $\mathscr{C}=\left\{c_{1}, c_{2}, c_{3}, \ldots, c_{d}\right\}$ with dimensions $p, q$ and $d$ respectively. Then $n=p \times q \times d$ different identification models are formed corresponding to the cross combinations of the grid points in the parameter space. Utilizing (1) the equations of motion corresponding to each model $I_{\zeta}$ can be represented as below

$$
I_{\zeta}:\left[\begin{array}{c}
\dot{\phi}_{\zeta} \\
\ddot{\phi}_{\zeta}
\end{array}\right]=\left[\begin{array}{cc}
0 & 1 \\
-\frac{k_{\zeta}-m g h_{\zeta}}{J_{x_{e q}}^{\zeta}}-\frac{c_{\zeta}}{J_{x_{e q}}^{\zeta}}
\end{array}\right]\left[\begin{array}{c}
\phi_{\zeta} \\
\dot{\phi}_{\zeta}
\end{array}\right]+\left[\begin{array}{c}
0 \\
\frac{m h_{\zeta}}{J_{x_{e q}}^{\zeta}}
\end{array}\right] a_{y},
$$

where $\zeta=1, \ldots, n$ denotes the identification model number and

$$
J_{x_{e q}}^{\zeta}=J_{x x}+m h_{\zeta}^{2}
$$

is the equivalent roll moment of inertia. We assume that all models have zero initial conditions such that $\phi_{\zeta}(0)=0$, and $\dot{\phi}_{\zeta}(0)=0$, for $\zeta=1, \ldots, n$. Note that the zero initial conditions physically correspond to starting the identification algorithm at a straight driving state, where the roll angle $\phi$, and the roll rate $\dot{\phi}$ of the vehicle are both zero. Also note that every model is driven by the same input $a_{y}$ (lateral acceleration), which is a measured sensor quantity of the vehicle.

We can then define the identification error for the $\zeta^{\text {th }}$ roll plane model as the difference between the vehicle's measured roll angle and the corresponding model output; we denote this by $e_{\zeta}$ and compute it from

$$
e_{\zeta}=\phi-\phi_{\zeta}, \quad \text { for } \quad \zeta=1,2, \ldots, n
$$

Next we compute the MMST cost function [7, 13] (a function of the identification error for each model)

$$
J_{\zeta}(t)=\alpha\left\|e_{\zeta}(t)\right\|+\beta \int_{0}^{t} e^{-\lambda_{f}(t-\tau)}\left\|e_{\zeta}(\tau)\right\| d \tau,
$$

where $\zeta=1, \ldots, n$ and $\alpha, \beta \geq 0$ are scalars controlling the relative weights on instantaneous and cumulative identification error measures. Also $\lambda_{f}$ denotes the forgetting factor. Switching among the models and choosing the one with the minimum cost based on the criterion below

$$
\eta(t)=\arg \min _{\zeta=1, \ldots, n} J_{\zeta}(t)
$$

yields the model with the minimum cumulative error and the selected model parameters $k_{\eta}, c_{\eta}$ and $h_{\eta}$, represent the vehicle in the parameter space described by $\mathscr{K}, \mathscr{C}$ and $\mathscr{H}$, respectively. Having described the controller switching criteria, we next give the procedure for designing individual rollover prevention controllers $\mathbf{C}_{\eta}$, for the switched controller implementation shown in Figure 3.

\subsection{Switched controllers based on differential brake input}

Here we present a simple proportional feedback control design for the individual switched controllers. The reason for using a P-controller design in this paper is twofold. Firstly, it is easier to implement and show the benefits of the suggested switched control design. Secondly, P-design has been used in the literature in the context of rollover prevention; for example see [6]. We emphasize that our suggested switched control approach can be used in conjunction with any other preferred control design method for the individual controllers. The switching in controller gains is determined by the realtime estimation of the CG height described earlier, where for each combination of $h_{\eta} \in\left\{h_{1}, \ldots, h_{p}\right\}, k_{\eta} \in\left\{k_{1}, \ldots, k_{q}\right\}$, and $c_{\eta} \in\left\{c_{1}, \ldots, c_{d}\right\}$, there is a paired local controller $\mathbf{C}_{\eta} \in\left\{\mathbf{C}_{\mathbf{1}}, \mathbf{C}_{\mathbf{2}}, \ldots, \mathbf{C}_{\mathbf{n}}\right\}$, where

$$
\mathbf{C}_{\eta}: \quad u_{\eta}=K_{\eta} a_{y}, \quad \eta \in\{1,2, \ldots, n\},
$$

which yields higher performance for the current values of $h_{\eta}$, $k_{\eta}, c_{\eta}$. We note that this controller switching rule is based on the certainty equivalence principle ${ }^{1}$. We further emphasize that the CG height and the lateral acceleration $\left(a_{y}\right)$ are the most important factors affecting rollover tendency of a vehicle. As we can estimate CG height, and $a_{y}$ is a measurable quantity, then one can use the simple feedback structure given in (11) for mitigating rollover.

We utilize the single track model with roll degree of freedom and with differential brake input given in (3) to design our simple proportional controllers. As $L T R_{d}$ is a metric directly related to rollover occurrence, we wish to synthesize a controller corresponding to each CG height setting, which prevents the peak value of $L T R_{d}$ below some prespecified level. Specifically, we want to keep

$$
\left\|L T R_{d}\right\| \leq 1
$$

for the largest possible steering inputs, which is equivalent to keeping all the 4 wheels in contact with the road and thus preventing rollover. The controller gains $K_{\eta}$ are chosen such that for a certain maximum speed $v_{\max }$ and a certain maximum steering input $\delta_{\max }(12)$ is satisfied. This in turn will guarantee that $\left\|L T R_{d}\right\| \leq 1$ for all $|\delta|<\left|\delta_{\max }\right|$ and $v<v_{\max }$ corresponding to the CG height $h_{\eta}$. In this respect each controller $C_{\eta}$ is a robust controller for the current CG height $h_{\eta}$.

Comment: A basic problem with the controller structure introduced above is that it is always active. That is, it will always attempt to limit the $L T R_{d}$, even in non-critical situations, thus potentially interfering with, and annoying the vehicle driver. It therefore makes sense to activate the controller in situations only when the potential for rollover is significant. One can limit this by putting a threshold output for the activation of the controllers. Since the system output considered here is the lateral acceleration we utilize the following rule for activating the switched controllers

$$
u=\left\{\begin{array}{lll}
u_{\eta} & \text { for } & \left|a_{y}\right| \geq\left[a_{y}\right]_{\text {threshold }} \\
0 & \text { for } & \left|a_{y}\right| \leq\left[a_{y}\right]_{\text {threshold }}
\end{array}\right.
$$

where $\left[a_{y}\right]_{\text {threshold }}$ is a positive scalar representing the controller activation limit. We note that the activation threshold depends

\footnotetext{
1 in the sense of adaptive control, the principle of certainty equivalence from tuning to switching is based on the hypothesis that a small identification error leads to a small tracking error [7],[15]. Therefore using a model that has the closest outputs to those of the plant is likely to yield the best feedback control performance.
} 
Table 2. Simulation model parameters

\begin{tabular}{cll}
\hline parameter & value & unit \\
\hline$m$ & 1300 & {$[\mathrm{~kg}]$} \\
$g$ & 9.81 & {$\left[\mathrm{~m} / \mathrm{s}^{2}\right]$} \\
$v$ & 40 (initial speed) & {$[\mathrm{m} / \mathrm{s}]$} \\
$\delta_{\text {peak }}$ & 90 & {$[\mathrm{deg}]$} \\
$J_{x x}$ & 400 & {$\left[\mathrm{~kg} \cdot \mathrm{m}^{2}\right]$} \\
$J_{z z}$ & 1200 & {$\left[\mathrm{~kg} \cdot \mathrm{m}^{2}\right]$} \\
$l_{v}$ & 1.2 & {$[\mathrm{~m}]$} \\
$l_{h}$ & 1.3 & {$[\mathrm{~m}]$} \\
$L$ & 2.5 & {$[\mathrm{~m}]$} \\
$T$ & 1.5 & {$[\mathrm{~m}]$} \\
$\mathbf{h}$ & $\mathbf{0 . 5}$ (initially unknown) & {$[\mathrm{m}]$} \\
$c$ & 5000 & {$\left[\mathrm{~kg} \cdot \mathrm{m}^{2} / \mathrm{s}\right]$} \\
$k$ & 36000 & {$\left[\mathrm{~kg} \cdot \mathrm{m}^{2} / \mathrm{s}^{2}\right]$} \\
$C_{v}$ & 60000 & {$[\mathrm{~N} / \mathrm{rad}]$} \\
$C_{h}$ & 90000 & {$[\mathrm{~N} / \mathrm{rad}]$} \\
\hline
\end{tabular}

on the vehicle type and parameters. In the following simulations $\left[a_{y}\right]_{\text {threshold }}=4 \mathrm{~m} / \mathrm{s}^{2}$ was utilized.

\section{NUMERICAL ANALYSIS}

In this section, we investigate the performance of the proposed adaptive switching rollover controller numerically. In the simulations, we have used the model parameters given in Table 2 for the model representing the simulated vehicle dynamics based on the single track model with roll degree of freedom. For ease of exposition of the numerical results, we assume that the suspension parameters $k$ and $c$ are fixed and known, but that the CG height is unknown. In doing so, we considered the CG height uncertainty to be such that $h \in \mathscr{H}=\{0.5,0.55, \ldots, 0.85\}$, comprising of 8 possible $\mathrm{CG}$ height configurations in total. Also we set the free design parameters for the cost function (9) as $\alpha=0.2$ and $\beta=0.8$, while the forgetting factor $\lambda_{f}$ was chosen to be 0 . We emphasize that the forgetting factor becomes important if the plant undergoes rapid switches; this is not the case for the CG height uncertainty considered here (where we assume that $\mathrm{CG}$ height is unknown and not changing in a finite time horizon), thus we set $\lambda_{f}=0$ in the following discussion.

For the design of local controllers, we assumed a peak vehicle speed of $v_{\max }=40[\mathrm{~m} / \mathrm{s}]$ (i.e. $124[\mathrm{~km} / \mathrm{h}]$ ), which represents typical freeway driving condition for a compact passenger vehicle. Furthermore, we assumed a peak steering wheel input of $\delta_{\max }=90^{\circ}$ (where the steering ratio of $1 / 18$ was assumed) to design the switched controllers such that, when the vehicle is operating at $\delta_{\max }$ and $v_{\max }$, the condition (12) satisfied for each CG height configuration, which is sufficient for mitigating rollover. We chose the controller gains $K_{\eta}$ as small as possible to minimize the control effort. Note that a feature of the control design suggested here is that it allows for reduction of controller actuation, which results in improved efficiency as compared to fixed robust control designs. The resulting 8 controller gains were calculated as follows

$$
\left.\begin{array}{ll}
K_{h=0.85}=-1280 \quad, & K_{h=0.80}=-1100 \\
K_{h=0.75}=-930 \quad, & K_{h=0.70}=-780 \\
K_{h=0.65}=-620 \quad, & K_{h=0.60}=-480 \\
K_{h=0.55}=-350 \quad, & K_{h=0.50}=-220
\end{array}\right\}
$$

In what follows, we present the simulation results corresponding to the switched control structure shown in Figure 3 that utilize the above control gains based on varying CG configurations. In our plots we provide comparisons of the switched
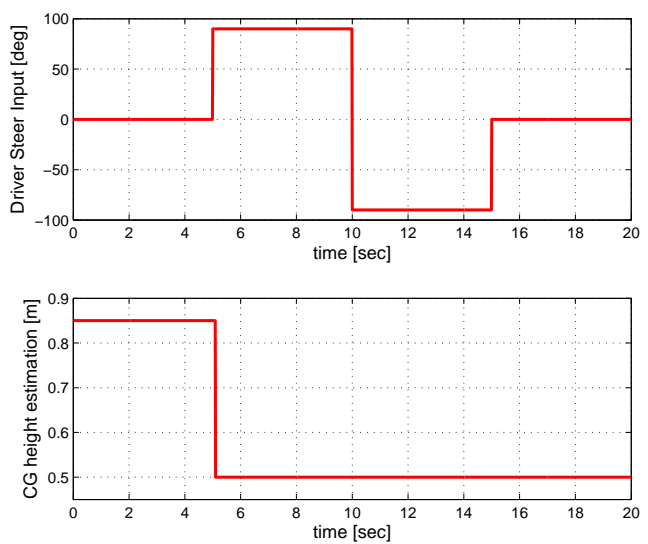

Fig. 4. Steering input and the resulting CG height estimation.

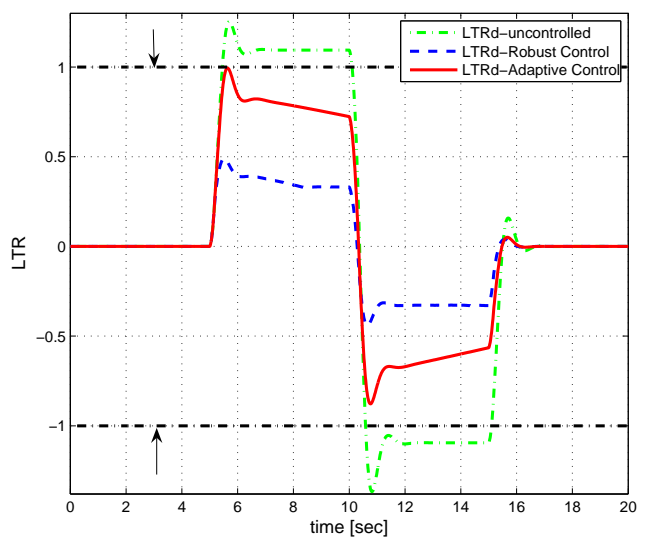

Fig. 5. Comparison of the $L T R_{d}$ for the controlled and uncontrolled vehicles.

adaptive controller with respect to a fixed robust controller, where the robust controller has the fixed gain $K_{h=0.85}$ assuming the worst case CG height of $h=0.85 \mathrm{~m}$. We also compare the results with uncontrolled single track model with roll degree of freedom, where appropriate.

For the numerical simulations, we used a typical obstacle avoidance maneuver known as the elk test with a peak driver steering input of magnitude $\delta_{\max }=90^{\circ}$ and with an initial speed of $v=124[\mathrm{~km} / \mathrm{h}]$. The steering profile corresponding to this maneuver and the resulting CG height estimation is shown in Figure 4 , where the worst case CG height (i.e., $h_{\max }=0.85[\mathrm{~m}]$ ) was assumed till the initiation of the steering maneuver. After the maneuver starts, the CG height was estimated to be $0.5[\mathrm{~m}]$ as seen from the figure. In Figure 5 we show the resulting $L T R_{d}$ comparisons for the controlled and the uncontrolled vehicles. Clearly the uncontrolled vehicle rolls over as $\left|L T R_{d}\right|>1$ during the maneuver. Also, both of the robust (i.e., fixed gain) and the switched adaptive controllers prevent rollover by keeping $\left|L T R_{d}\right|<1$. However, the adaptive controller does it in a less conservative way which is favorable. In Figure 6 we compare the vehicle states of the controlled and the uncontrolled vehicles, where we observe that due to smaller attenuation obtained by the adaptive (switched) controller, the resulting states trajectories are closer to the uncontrolled vehicle states as compared to the robust one. Again, this is favorable as the adaptive controller causes smaller driver intervention, and maintains a 


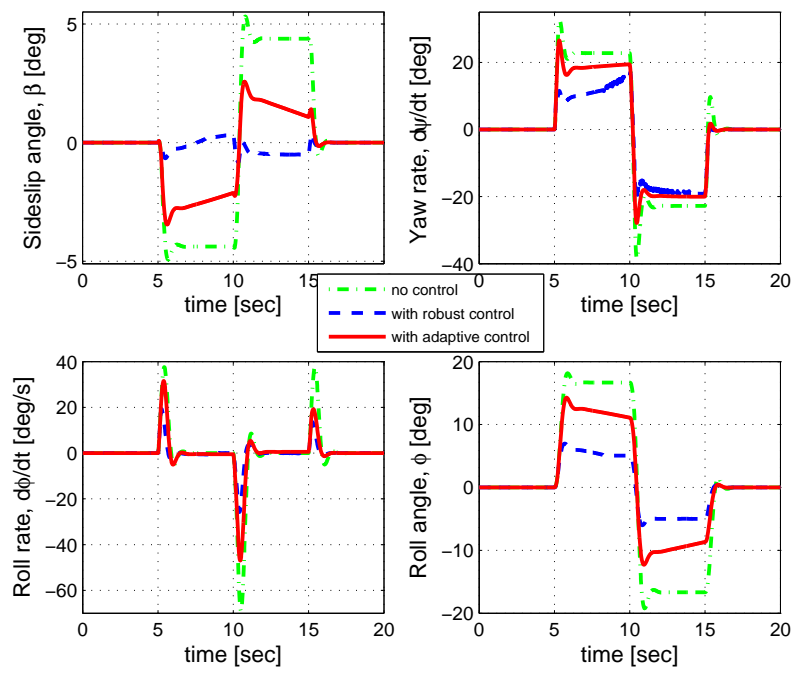

Fig. 6. Comparison of the vehicle states for the controlled and uncontrolled vehicles.
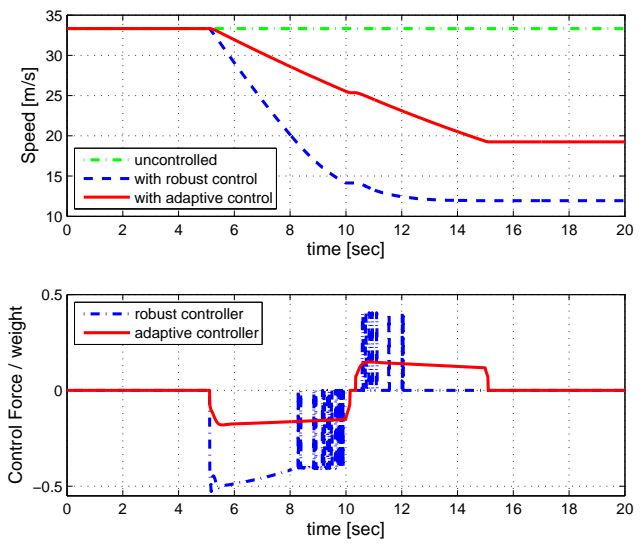

Fig. 7. Comparison of the vehicle speeds and the normalized control force.

natural response of the vehicle. Finally, in Figure 7 we show the vehicle speed and the normalized braking force variations for the controlled and the uncontrolled vehicles. We observe that the adaptive controller results in much less controller actuation and less drop in vehicle speed; this clearly shows the performance benefit of using the suggested switched controller as compared to the fixed robust control alternative.

\section{CONCLUSION}

In this paper, we presented a switched adaptive vehicle rollover prevention control design using differential braking actuators. We suggested the use of real time estimation of CG height as a controller switching criteria. We demonstrated our control designs with numerical simulations and compared the results with fixed robust controllers. The results indicate significant performance gains over the robust controller alternatives. We emphasize that due to the chosen control actuator (i.e., differential braking), our suggested control designs can easily be implemented and tested without much financial overhead, since these actuators already exist in most stock passenger vehicles.
Future work will proceed in several directions. We will explore the control scheme with respect to transients, as the rollover problem is a safety critical problem. Particularly, we are interested in the effect of sudden load changes during rollover, and we will investigate the controller selection criteria to mitigate the transients as an integral part of our control design. As part of this, we shall also look into alternative designs for individual switched controllers. Also, on the practical side of this work, we wish to implement and test the controller on a test vehicle with an industrial partner.

\section{REFERENCES}

National Highway Trafic Safety Administration (NHTSA), Trafic Safety Facts 2004: A Compilation of Motor Vehicle Crash Data from the Fatality Analysis Reporting System and the General Estimates System,Technical Report, 2006.

Carlson C.R. and Gerdes J.C., "Optimal Rollover Prevention with Steer by Wire and Differential Braking", Proc. of ASME Int. Mech. Eng. Congress and Exposition,Washington D.C.,November 1621,2003.

Akar M., Solmaz S. and Shorten R., 2006, Method for Determining the Center of Gravity for an Automotive Vehicle, Irish Patent Ref: (S2006/0162).

Solmaz S., Akar M., Shorten R., "Online Center of Gravity Estimation in Automotive Vehicles using Multiple Models and Switching", 9th IEEE Int. Conf. on Control, Automation, Robotics and Vision, Singapore, Dec 5-8, 2006.

S. Solmaz, M. Akar, R. Shorten, and J. Kalkkuhl, "Realtime Multiple-Model Estimation of Center of Gravity Position in Automotive Vehicles", Vehicle System Dynamics Journal, To appear, 2007.

Chen B. and Peng H., "Differential-braking-based rollover prevention for sport utility vehicles with human-in-the-loop evaluations", Vehicle System Dynamics, Vol. 36, pp. 359-389, 2001.

Narendra K.S., Balakrishnan J. and Ciliz K., 1995, Adaptation and Learning Using Multiple Models, Switching and Tuning. IEEE Control Systems, No. 0272-1708/95.

U. Kiencke, and L. Nielsen, Automotive Control Systems for Engine, Driveline and Vehicle, Springer-Verlag \& SAE Int., Berlin, 2000.

Selim Solmaz, Topics in Automotive Rollover Prevention: Robust and Adaptive Switching Strategies for Estimation and Control. $\mathrm{PhD}$ thesis, Hamilton Institute, Nat. Univ. of Ireland-Maynooth, 2007.

Odenthal D., Bünte T. and Ackermann J., "Nonlinear steering and braking control for vehicle rollover avoidance", Proc. of European Control Conference, Karlsruhe, Germany, 1999.

S. Solmaz, M. Corless, and R. Shorten, "A Methodology for the Design of Robust Rollover Prevention Controllers for Automotive Vehicles: Part 1-Differential Braking", Conf. on Decision and Control, San Diego, USA, 13-15 Dec. 2006.

S. Solmaz, M. Corless, and R. Shorten, "A Methodology for the Design of Robust Rollover Prevention Controllers for Automotive Vehicles: Part 2-Active Steering", American Control Conference, New York, USA, July 11-13, 2007.

Narendra K.S. and Balakrishnan J., "Improving Transient Response of Adaptive Control Systems using Multiple Models and Switching", Conference on Decision and Control, San Antonio, Texas, 1993

Narendra K.S. and Balakrishnan J., 1994, Improving Transient Response of Adaptive Control Systems using Multiple Models and Switching. IEEE Transactions on Automatic Control, Vol. 39, No. 9.

Narendra K.S., and Balakrishnan J., 1997, Adaptive Control using Multiple Models. IEEE Transactions on Automatic Control, Vol.42, No.2, pp 171-187. 